\title{
Utilização de Padrões no Ciclo de Vida de Aplicações de Aprendizagem: Um Mapeamento Sistemático
}

\author{
Maria Lydia Fioravanti, Ranieri de Brito Moreira, Ellen Francine Barbosa \\ ${ }^{1}$ Instituto de Ciências Matemáticas e de Computação (ICMC) \\ Universidade de São Paulo (USP) - São Carlos/SP - Brasil \\ \{mlfioravanti, ranieribm\}@usp.br, francine@icmc.usp.br
}

\begin{abstract}
The evolution of mobile computing outlined a new setting for teaching and learning applications. In this scenario, the existing educational applications, despite having several benefits and facilities, present problems and challenges that need to be explored. In software reuse field, we have observed a growing adoption of patterns, which allow using a reusable general solution to a problem which occurs frequently. This study aims at the identification of studies showing the use of patterns during learning applications lifecycle. Thus, in this work we conducted a systematic mapping in which 37 scientific articles were analyzed. The results are relevant to outline the current landscape of the area and they also highlight discussions that may guide future investigations in m-learning and t-learning, considering educational and pedagogical aspects.
\end{abstract}

Resumo. A evolução da computação móvel delineou um novo cenário para as aplicações de ensino e aprendizado. Nesse cenário, as aplicações educacionais existentes, mesmo possuindo diversos benefícios e facilidades, apresentam problemas e desafios que precisam ser explorados. Na área de reúso de software, tem-se percebido uma crescente adoção da abordagem de padrões, que possibilita a utilização de uma solução geral reutilizável para um problema que ocorre com frequência. Este estudo visa a identificação de estudos que mostrem a utilização de padrões no ciclo de vida de aplicações de aprendizagem. Para atingir o objetivo, foi conduzido um mapeamento sistemático, no qual foram estudados 37 artigos científicos. Os resultados obtidos são relevantes por destacarem o panorama atual da área, bem como por apresentarem discussões que podem nortear investigações futuras paras as modalidades m-learning $e$ t-learning, considerando aspectos educacionais e pedagógicos.

\section{Introdução}

Nos últimos anos, temas relacionados ao ensino e aprendizagem têm sido cada vez mais discutidos e estudados pela comunidade científica. Em especial, ambientes computacionais de aprendizagem vêm apresentando uma crescente importância, tendo um papel fundamental em atividades de ensino e treinamento, sendo relevantes não apenas no âmbito acadêmico como também no meio industrial [Barbosa 2004, Svetlana and Yonglk-Yoon 2009].

Esse cenário, associado ao rápido crescimento da adoção de tecnologias de informação e comunicação, tem favorecido o surgimento de novas modalidades de ensino, proporcionando novos meios de abordar as deficiências do ensino tradicional. $\mathrm{O}$ 
electronic learning (e-learning) ou aprendizagem eletrônica é a modalidade de ensino apoiada por tecnologia [Keegan 2005], que oferece mecanismos para o ensino baseados em computadores e tecnologias de rede.

A crescente adesão ao $e$-learning juntamente com o advento e desenvolvimento da computação ubíqua, vêm fomentando o surgimento de outras modalidades de ensino - a aprendizagem móvel ou mobile learning (m-learning) [Kinshuk et al. 2003, Nah et al. 2008, O'Malley et al. 2003, Wexler et al. 2008]; e a aprendizagem baseada em TV digital interativa ( $t$-learning). $\mathrm{O}$ m-learning se caracteriza pela capacidade de proporcionar uma forte interação entre os aprendizes e instrutores, possibilitando aos mesmos contribuir, participar e acessar o ambiente de ensino por meio de dispositivos móveis (celulares, tablets, PDAs, entre outros) a qualquer momento e em qualquer lugar. Já o $t$ learning promove o ambiente de aprendizagem proporcionado pela plataforma televisiva.

Apesar dos benefícios ao ensino e aprendizagem, por serem conceitos novos e ainda incipientes, o m-learning e o t-learning apresentam algumas limitações em sua construção e utilização, tais como [Quinta and de Lucena 2010]: tela de tamanho variável (às vezes monocromática); quantidade de cores suportadas; poder de processamento reduzido; baixa capacidade de armazenamento; energia limitada (dependente de baterias nos dispositivos móveis); mecanismos de interação limitados; baixa largura de banda e alto custo de planos de dados para internet; falta de padronização no suporte a mídia dos aparelhos; aspectos de usabilidade e de acessibilidade; e carência de soluções que promovam e estimulem o reúso e a interoperabilidade dos recursos educacionais que tanto diminuem os custos quanto possibilitam a melhoria da qualidade dos sistemas gerados.

Diante desse contexto, padrões de software podem surgir como uma ferramenta capaz de auxiliar os engenheiros de software e desenvolvedores na construção de aplicações de ensino e aprendizagem.

A reutilização de software é um propósito enunciado quase que simultaneamente com o surgimento da própria Engenharia de Software [Bosch et al. 1999]. Com o objetivo de reúso em diferentes níveis de abstração, surgiram na década de 90 os padrões de software [Buschmann et al. 1996, Coad 1992, Coplien 1992, Gamma et al. 1995], que tentam captar a experiência adquirida no desenvolvimento de software e sintetizá-la em forma de problema e solução. Além disso, ajudam a melhorar a comunicação entre os desenvolvedores, que podem conduzir suas discussões com base nos nomes dos padrões [Gamma et al. 1995]. De acordo com Appleton [Appleton 1997], os componentes essenciais que devem ser claramente identificáveis ao se ler um padrão são: nome, problema, contexto, forças, solução, exemplos, padrões relacionados e utilizações conhecidas.

Este trabalho tem como objetivo caracterizar o cenário atual da utilização de padrões em aplicações de aprendizagem e-learning, m-learning e t-learning a partir da condução de um Mapeamento Sistemático [Kitchenham 2004]. A ideia é que os resultados forneçam evidências para a proposição de um conjunto de padrões para novas modalidades de ensino, ou seja, o objetivo é demonstrar se há deficiência de estudos nessas áreas de pesquisa bem como as lacunas existentes para a condução de novas pesquisas.

O restante do artigo está organizado como se segue. Na Seção 2 é apresentado o Mapeamento Sistemático conduzido no contexto de utilização de padrões no ciclo de vida de aplicações de aprendizagem. Na Seção 3 são apresentados os dados extraídos e 
sumarizados. As evidências encontradas como respostas às questões de pesquisa definidas no protocolo do Mapeamento Sistemático são discutidas na Seção 4. Por fim, na Seção 5 são apresentadas as conclusões e perspectivas para a continuidade do trabalho.

\section{Mapeamento Sistemático: Visão Geral}

Mapeamento Sistemático (Systematic Mapping - SM) é um tipo de estudo secundário que fornece um processo pelo qual se realiza uma revisão mais ampla dos estudos primários e cujo objetivo é, a partir dos estudos primários relevantes descobertos, obter uma visão ampla da área de pesquisa. Desse modo, pode-se identificar evidências disponíveis, bem como identificar lacunas no conjunto dos estudos primários e áreas nas quais mais estudos primários precisam ser conduzidos [Kitchenham 2004].

A condução desse mapeamento sistemático segue as diretrizes propostas na literatura por Kitchenham e Charters [Kitchenham and Charters 2007].

\subsection{Planejamento}

Esse mapeamento sistemático tem como objetivo identificar, classificar e analisar estudos que contemplam a utilização de padrões ao longo do ciclo de vida de aplicações de aprendizagem eletrônica. Além disso, apresentar uma análise sobre os estudos recuperados fornecendo evidências para a proposição de um conjunto de padrões para os segmentos m-learning e t-learning. Assim, o objetivo é demonstrar se há deficiência de estudos nessas áreas de pesquisa e as lacunas existentes para a condução de novas pesquisas.

Para atingir os objetivos estabelecidos, foi definido um protocolo para a condução do mapeamento, disponível integralmente em http: / / goo.gl / 4 jxF jM, no qual foram estabelecidas as questões de pesquisa que nortearam o trabalho.

QP1: Quais padrões são aplicados ao longo do ciclo de vida de aplicações e-learning?

QP2: Quais padrões são aplicados ao longo do ciclo de vida de aplicações m-learning?

QP3: Quais padrões são aplicados ao longo do ciclo de vida de aplicações t-learning?

Neste mapeamento as buscas foram realizadas em bases eletrônicas com maior relevância para a área da computação, ou seja, nas bases que indexam grande quantidade de artigos relacionados a diferentes conferências e revistas da área de interesse do mapeamento: Scopus, Web of Science, IEEE Xplore, ACM Digital Library, Science Direct.

Um passo importante para realização das buscas automáticas é a definição da string de busca. Para esse mapeamento a string foi definida pela junção dos termos de maior relevância para pesquisa. Os termos da busca, e os sinônimos desses termos, foram definidos de acordo com a opinião de especialistas, de acordo com a literatura e com as questões de pesquisa definidas para o mapeamento.

Os principais termos utilizados na condução do mapeamento estão relacionados com padrões (pattern) e com os tipos de aplicações de aprendizagem eletrônica, ou seja, $e$ learning, m-learning e $t$-learning. Essas palavras-chave e seus sinônimos foram utilizados na elaboração da string de busca apresentada a seguir.

Após a definição da string de busca genérica, foram definidos critérios de apoio para a etapa de seleção da fase de condução do mapeamento. A definição desses critérios tem como objetivo selecionar, dentre os trabalhos resultantes da busca automática, aqueles que têm potencial para responder às questões de pesquisa elaboradas e que tenham 
relação direta com o assunto pesquisado. Os critérios de inclusão e exclusão são essenciais para o processo de seleção de um mapeamento e devem ser definidos ainda na fase de planejamento, uma vez que não devem sofrer nenhum tipo de viés com relação ao resultado das buscas automáticas.

Os artigos foram categorizados conforme os seguintes critérios de inclusão (CI):

CI.1. Estudos primários que apresentam a proposição ou utilização de um padrão no contexto de e-learning.

CI.2. Estudos primários que apresentam a proposição ou utilização de um padrão no contexto de m-learning.

CI.3. Estudos primários que apresentam a proposição ou utilização de um padrão no contexto de t-learning.

Visando eliminar estudos irrelevantes para as questões de pesquisa, os seguintes critérios de exclusão foram adotados: artigos que não envolviam o tema das questões de pesquisa, artigos que não estavam escritos na língua inglesa ou portuguesa, artigos duplicados, indisponíveis para download, artigos anteriores à 1990 (época da emersão de padrões no contexto da computação), artigos de áreas que não fossem engenharia ou computação e artigos que não foram publicados em periódicos ou anais de conferências.

\subsection{Condução}

A fase de condução do mapeamento foca em fornecer um conjunto de trabalhos primários que possam contribuir para o mapeamento, respondendo parcialmente ou integralmente as questões de pesquisa estabelecidas. Essa fase está dividida em três etapas principais.

\subsubsection{Busca nas bases eletrônicas e exclusão dos trabalhos duplicados}

A primeira etapa da condução de um mapeamento é a realização das buscas por trabalhos primários nas bases eletrônicas selecionadas. Inicialmente, foi realizado o ajuste da string de busca genérica para cada uma das bases selecionadas de acordo com as características e especificidades das mesmas. Em seguida, foram realizadas as buscas em cada uma das bases, aplicando-se os filtros por data e área de interesse. Por fim, foi efetuado o download, em cada uma das bases, das referências dos artigos resultantes da busca.

As buscas automáticas retornaram um total de 1589 estudos, sendo que 470 foram eliminados por serem trabalhos duplicados, juntamente com os 10 arquivos de divulgação de conferências, restando 1109 estudos selecionados. Conforme pode ser visto na Figura 1, em que são ilustradas as três etapas da condução, a busca na Scopus retornou 802 estudos; na Web of Science, 472; na IEEE, 238; na ACM, 45; e na Science Direct, 32.

A base eletrônica Scopus apresentou o maior número de trabalhos retornados, totalizando 50\% dos resultados, uma vez que indexa a maioria dos trabalhos relevantes da área, além de conferências que também são contempladas por outras bases. Já as bases 
CBIE-LACLO 2015

Anais do XXVI Simpósio Brasileiro de Informática na Educação (SBIE 2015)

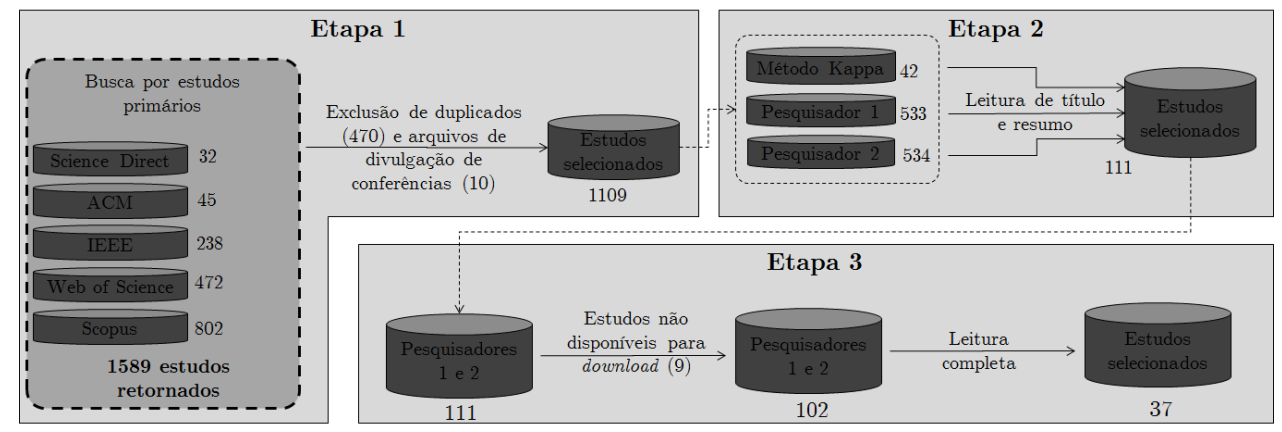

Figura 1. Etapas do mapeamento sistemático.

Web of Science, IEEE, ACM e Science Direct tiveram 30\%, 15\%, 3\% e 2\%, respectivamente, do total de resultados. Vale ressaltar que essa primeira etapa da condução foi realizada colaborativamente por dois pesquisadores.

\subsubsection{Seleção de trabalhos por títulos e resumos}

Essa etapa do estudo consistiu em aplicar os critérios de inclusão e exclusão nos trabalhos que foram selecionados na etapa anterior. A partir da leitura dos títulos e resumos de todos os trabalhos, os autores decidiram se esses trabalhos seriam incluídos para a próxima etapa ou não. Cada um dos pesquisadores foi responsável pela seleção de metade dos trabalhos. Em caso de dúvida o outro pesquisador era consultado e ambos chegavam a um consenso. Permanecendo a dúvida, o trabalho era incluído para análise mais detalhada.

Para saber se uma dada classificação de um objeto é confiável, é necessário ter este objeto classificado várias vezes, por exemplo, por mais de um juiz [Fleiss 1981]. Neste cenário, pode-se utilizar a medida Kappa para obter a intensidade da concordância entre dois ou mais juízes, uma vez que esta é baseada no número de respostas concordantes, ou seja, no número de casos cujo resultado é o mesmo entre os juízes. O Kappa é uma medida cujo valor máximo é 1 , sendo que este valor representa total concordância. As interpretações dos demais valores de Kappa são: (i) < 0 não há concordância; (ii) 0-0.19 há concordância fraca; (iii) 0.20-0.39 há concordância justa; (iv) 0.40-0.59 há concordância moderada; (v) 0.60-0.79 há concordância substancial e (vi) 0.80-1.00 há concordância quase perfeita.

Para garantir sintonia entre os pesquisadores e agregar confiabilidade ao processo de seleção, foi realizada a classificação de 21 estudos selecionados arbitrariamente para obter a medida Kappa. Na Tabela 1(a) são apresentados os dados da análise dos 21 trabalhos selecionados pelos juízes A e B com a decisão de incluir (I) ou excluir (E) o trabalho.

O valor Kappa geral calculado com os resultados demonstrados na Tabela 1(a) indicaram uma concordância substancial entre os pesquisadores com valor de 0,618 . Devido ao valor estar muito perto da faixa de concordância moderada, os pesquisadores optaram por analisar caso a caso as discordâncias e refazer a medição com outros 21 trabalhos após discussão sobre os pontos divergentes. Na Tabela 1(b) são apresentados os dados da análise dos outros 21 trabalhos selecionados.

O valor Kappa geral calculado com os resultados demonstrados na Tabela 1(b) teve um aumento e passou para 0,774 , o que ainda indica uma concordância substancial, 
Tabela 1. Dados utilizados no cálculo do método Kappa.

(a) Medição 1

\begin{tabular}{|cc|cc|c|}
\hline & & \multicolumn{2}{|c|}{ B } & \\
& & I & E & Total \\
\hline \multirow{2}{*}{ A } & I & 8 & 2 & 10 \\
& E & 2 & 9 & 11 \\
\hline \multicolumn{2}{|c|}{ Total } & 10 & 11 & 21 \\
\hline
\end{tabular}

(b) Medição 2

\begin{tabular}{|cc|cc|c|}
\hline & & \multicolumn{2}{|c|}{ B } & \\
& & I & E & Total \\
\hline \multirow{2}{*}{ A } & I & 2 & 1 & 3 \\
& E & 0 & 18 & 18 \\
\hline \multicolumn{2}{|c|}{ Total } & 2 & 19 & 21 \\
\hline
\end{tabular}

porém muito mais próxima da concordância quase perfeita. Vale ressaltar que houve somente uma classificação divergente. Devido ao tamanho da amostra, a concordância quase perfeita só seria atingida se não houvesse nenhuma discordância entre os juízes e, então, os pesquisadores entenderam que esse resultado mostrava-se satisfatório para garantir confiabilidade na seleção dos estudos.

Dos 1109 estudos selecionados, 42 foram utilizados no método Kappa para cálculo de concordância e os demais foram divididos entre os pesquisadores para seleção. Assim, um dos pesquisadores efetuou a seleção de 533 estudos e o outro de 534. Dentre os 1109 estudos analisados, 111 foram selecionados para a próxima etapa da execução.

\subsubsection{Seleção de trabalhos por leitura na íntegra}

A última etapa da condução do mapeamento foi a leitura integral dos 111 trabalhos a procura de elementos que pudessem responder às questões de pesquisa definidas. Nessa etapa, 9 dos estudos não foram encontrados para download, sendo assim excluídos por indisponibilidade. Os 102 trabalhos restantes foram lidos integralmente por ambos os pesquisadores e, em consenso, foram selecionados como relevantes ou não. Dentre os 102 artigos lidos, 37 foram selecionados para etapa de extração e sumarização dos dados.

Durante a leitura integral dos trabalhos, os trabalhos que não eram excluídos foram analisados e algumas informações foram extraídas. A próxima fase do mapeamento foi conduzida com base nessas informações.

\subsection{Extração e Sumarização dos dados}

Nessa etapa os dados dos trabalhos primários foram extraídos e sumarizados com o intuito de responder às questões de pesquisa. O primeiro passo dessa etapa foi a extração dos seguintes dados: Título, Autor(es), País (para cada autor), Fonte (conferência ou periódico), Tipo de aprendizagem eletrônica (e-learning, m-learning ou t-learning), Classificação do padrão, e Nome do padrão. Tal extração foi realizada durante a leitura integral dos 37 trabalhos finais selecionados.

O segundo passo foi a sumarização dos dados, isto é, os dados dos trabalhos foram reunidos com o objetivo de responder às questões de pesquisa. A partir da etapa de sumarização foi possível realizar algumas observações sobre os trabalhos selecionados.

\section{Resultados da Extração de Dados}

Entre os 37 estudos recuperados, pode-se perceber que não há uma distribuição padrão por ano, como ilustrado na Figura 2, que apresenta a quantidade de estudos por ano de publicação. Nota-se, entretanto, uma tendência sutil de maior exploração do tema entre 
2009 e 2013. Considerando que essa é uma área não tão consolidada, em que os primeiros estudos recuperados são de 2003, há indícios de que tem sido pouco explorada, embora haja potencial para tal. No que tange aos veículos de publicação, na Figura 3 observa-se que há 33 estudos de conferências e somente 4 de periódicos, o que pode indicar certa imaturidade da área de pesquisa.

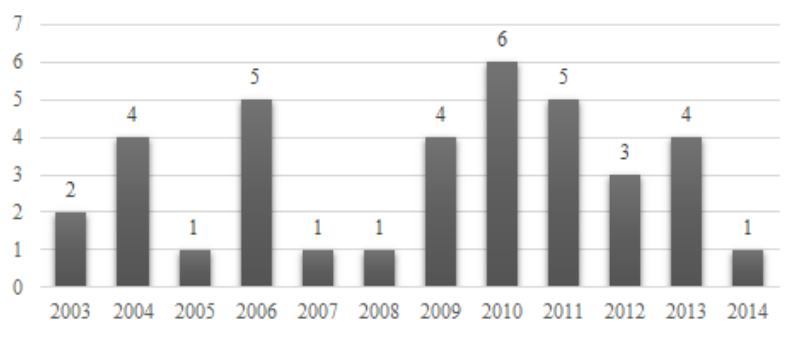

Figura 2. Quantidade de estudos por ano de publicação.

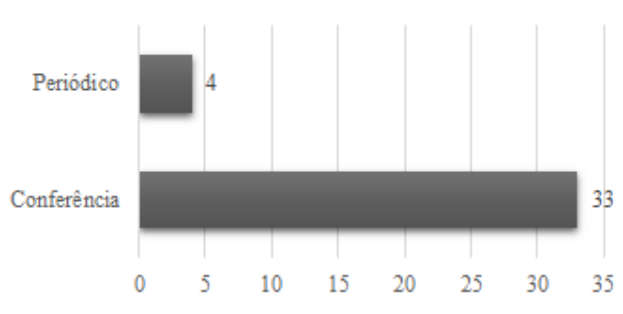

Figura 3. Quantidade de estudos por ano de publicação.

Classificando os estudos recuperados com relação à modalidade de ensino e aprendizagem, ilustrado na Figura 4, foi possível concluir que o segmento de e-learning já tem sido bastante explorado, sendo que $86,49 \%$ dos estudos recuperados tratam sobre a utilização de padrões nessa modalidade. No entanto, o segmento de $m$-learning necessita de mais iniciativas de pesquisa, bem como t-learning, que não foi contemplado em nenhum estudo recuperado.

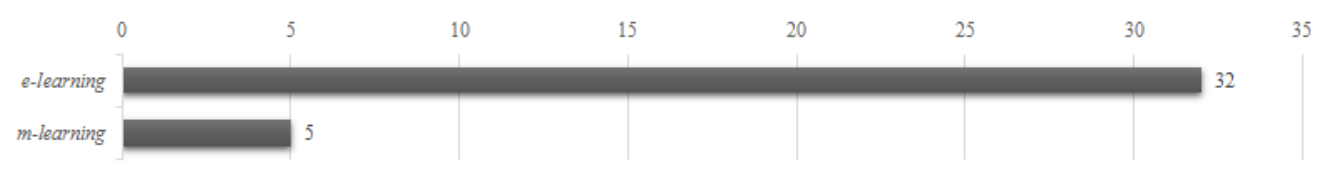

Figura 4. Modalidade de Ensino e Aprendizagem.

\section{Análise e Discussão dos Resultados}

As principais discussões apresentadas a seguir são fundamentadas nos resultados obtidos a partir da análise dos 37 estudos primários identificados e fornecem respostas para as questões de pesquisa definidas. As referências dos estudos recuperados estão disponíveis em http://goo.gl/OKI zOM.

A partir do mapeamento sistemático conduzido foi possível identificar diversos padrões aplicados ao longo do ciclo de vida de aplicações e-learning e m-learning. No entanto, nem todos os estudos apresentavam os componentes essenciais dos padrões [Appleton 1997]. Com base no que foi definido na fase de extração e sumarização de dados, para responder à questão de pesquisa seria necessário o nome e a classificação do padrão apresentado, mas na maioria dos estudos recuperados isso não foi possível.

De posse das informações que puderam ser extraídas dos estudos, na Tabela 2 pode ser observada a quantidade de padrões encontrados para cada classe identificada.

Na Figura 4 já havia sido constatado que os padrões não são explorados no contexto de t-learning. No entanto, sem a análise dos estudos recuperados não se tinha delineado o panorama das demais modalidades.

As únicas classes de padrões que apareceram tanto em e-learning quanto em $m$-learning foram os padrões arquiteturais, que expressam formas de organizar a estru- 


\begin{tabular}{lccc}
\multicolumn{4}{c}{ Tabela 2. Quantidade de Padrões por Modalidade de Ensino. } \\
\hline Padrão & e-learning & m-learning & t-learning \\
\hline Padrões arquiteturais & 4 & 2 & 0 \\
Design Patterns & 21 & 10 & 0 \\
Padrões de comunicação & 2 & 0 & 0 \\
Padrões de visualização & 0 & 1 & 0 \\
Padrões conceituais & 1 & 0 & 0 \\
Padrões para o domínio educacional & 69 & 0 & 0 \\
\hline
\end{tabular}

tura fundamental do sistema, e os design patterns, que visam facilitar a reutilização de soluções na fase de projeto do software.

\subsection{QP1: Quais padrões são aplicados ao longo do ciclo de vida de aplicações e-learning?}

De um total de 32 estudos selecionados, 12 referem-se à utilização de padrões arquiteturais, como por exemplo o Model-View-Controller (MVC). Como observado na Tabela 2, outra classe de padrões que aparece com frequência, totalizando 21 padrões, são os Design Patterns, tais como Composite, Abstract Factory ou ainda outros padrões GoF e J2EE. Em alguns casos, o termo é utilizado como sinônimo de padrão de software ou de padrões de maneira geral, enquanto em outros, visto que o nome do padrão não é apresentado, não se sabe ao certo qual o tipo de padrão que o estudo trata. Foram identificados, ainda, padrões de comunicação e conceitual no contexto de $e$-learning.

Além desses padrões cujo objetivo é tratar os problemas de implementação da aplicação, considerando sua arquitetura ou ainda a orientação a objetos, foram identificadas famílias de padrões para o domínio de aplicações de aprendizagem. Padrões pedagógicos, padrões para aprendizado colaborativo e padrões de avaliação são exemplos de famílias de padrões que subsidiam a construção de aplicações educacionais. Alguns exemplos dos 69 padrões encontrados pertencentes a estas famílias são Content authoring, Video lecture, Personalization, E-book delivery e Glossary.

Em um dos estudos recuperados, Avgeriou et al. [Avgeriou et al. 2003] propõem uma linguagem de padrões. O padrão que foi nomeado Personalization, apresentado pelos autores, é um exemplo que contém alguns dos componentes recomendados por Appleton [Appleton 1997]. O problema levantado é como organizar os diversos cursos de que um usuário participa para que seja apresentado a ele seu conjunto personalizado de cursos. A motivação apresentada é o fato de um usuário estar envolvido com diversos cursos a cada semestre, sendo que em cada um deles pode atuar de maneira diferente (tutor, aluno, etc). Em seguida, é apresentada a solução que sugere criar um serviço de personalização para todos os usuários, que personaliza a sua home page de acordo com uma conta única pela qual é efetuado o acesso e é possível identificar os cursos em que o usuário em questão está envolvido. Além disso, os autores apresentam Categoria de usuário, Usos Conhecidos e Padrões Relacionados.

Conforme evidenciado na Tabela 2, os padrões para aplicações e-learning são bem explorados tanto no que diz respeito a padrões de domínio específico (educacional) quanto nas classes de padrões que guiam detalhes técnicos da construção de aplicações de aprendizagem. No entanto, somente 11 estudos tratam de padrões específicos para o domínio educacional, evidenciando que embora o assunto já seja tema de investigação, 
ainda é carente de pesquisas mais profundas que abordem os aspectos educacionais e pedagógicos na criação das aplicações.

\subsection{QP2: Quais padrões são aplicados ao longo do ciclo de vida de aplicações m-learning?}

De um total de 5 estudos, 3 deles citaram a utilização de dois padrões arquiteturais diferentes, sendo que 2 deles abordam a utilização do padrão MVC. Outra classe de padrões citada em 2 estudos é a dos Design Patterns, sendo que 10 deles foram mencionados. Um dos estudos cita, ainda, a utilização de padrões de visualização, que nesse contexto são padrões para selecionar a melhor técnica de visualização para a interface.

Pode-se notar que o cenário da utilização de padrões no contexto de m-learning, diferentemente do de e-learning, tem sido pouco explorado. Os estudos selecionados não focam na proposição ou utilização de um conjunto de padrões, mas sim utilizam padrões já consolidados para a construção de suas aplicações. Vale ressaltar, ainda, que os padrões elencados para aplicações móveis de aprendizagem não são padrões de domínio específico, mas sim padrões arquiteturais e padrões de projetos geralmente utilizados em aplicações construídas utilizando o paradigma de orientação a objetos.

De acordo com o constatado na Tabela 2, padrões voltados ao contexto educacional ainda não têm sido explorados. Portanto, é possível observar pontos que podem ser mais bem explorados considerando o domínio de aprendizagem móvel, como por exemplo padrões que guiem a solução do problema de telas de tamanho variável, ou ainda, poder de processamento reduzido.

\subsection{QP3: Quais padrões são aplicados ao longo do ciclo de vida de aplicações t-learning?}

Conforme já mencionado, nenhum estudo recuperado trata da utilização de padrões em aplicações de TV digital interativa. Portanto, é possível observar uma lacuna na área de pesquisa dessa nova modalidade de ensino, que vêm crescendo ao longo dos últimos anos.

\section{Conclusões e Trabalhos Futuros}

Este trabalho teve por objetivo analisar estudos que contemplam a utilização de padrões em aplicações de aprendizagem eletrônica por meio de um processo de mapeamento sistemático. O mapeamento sistemático se mostrou uma metodologia eficaz, embora dispendiosa de tempo, que envolveu um trabalho árduo de leitura e análise dos estudos primários a fim de se obter respostas às questões levantadas para a pesquisa.

Os 37 trabalhos selecionados contribuíram para formalizar o estado da arte com relação aos padrões no contexto de aprendizagem eletrônica. No que diz respeito ao benefício acadêmico, os levantamentos apontaram que a utilização de padrões para aplicações de aprendizagem móvel e TV digital interativa ainda são pouco explorados e podem agregar diversos benefícios relacionados ao reúso.

Como trabalhos futuros, com base nos resultados obtidos, aponta-se a exploração da utilização de padrões nas modalidades de ensino m-learning e $t$-learning. Os padrões de desenvolvimento podem ser mais bem explorados para tratar os desafios impostos pelas características intrínsecas aos dispositivos móveis, tais como tamanho de tela variável ou baixa largura de banda. 
No entanto, os desafios associados a aprendizagem móvel e de TV digital não se limitam a aspectos de desenvolvimento ou tecnologias. Deve-se considerar, ainda, os pontos de vista educacional e pedagógico: manter o aprendiz motivado evitando evasão, tratar diferentes estilos de aprendizagem (visual, lógico, social, etc.), guiar o aprendiz no autodidatismo, entre outras especificidades que podem ser cruciais no projeto de uma aplicação de aprendizagem destas modalidades. Neste cenário, padrões são ferramentas de extrema importância para orientar os projetistas e desenvolvedores deste tipo de aplicação, contribuindo tanto para evitar os problemas já conhecidos sem ter que redescobri-los quanto para agregar qualidade ao software por se tratarem de soluções bem sucedidas.

\section{Agradecimentos}

Os autores agradecem o apoio financeiro da FAPESP e CAPES.

\section{Referências}

Appleton, B. (1997). Patterns and software: Essential concepts and terminology.

Avgeriou, P., Papasalouros, A., Retalis, S., and Skordalakis, M. (2003). Towards a pattern language for learning management systems. Educational Technology \& Society, 6(2):11-24.

Barbosa, E. F. (2004). Uma Contribuição ao Processo de Desenvolvimento e Modelagem de Módulos Educacionais. Tese de doutorado, Instituto de Ciências Matemáticas e de Computação - ICMC/USP, São Carlos, SP.

Bosch, J., Molin, P., Mattson, M., and Bengtsson, P. (1999). Framework Problems and Experiences. In Fayad, M., Schmidt, D., and Johnson, R., editors, Building Application Frameworks - Object Oriented Foundations of Framework Design, pages 55-82. John Willey and Sons.

Buschmann, F., Meunier, R., Rohnert, H., Sommerlad, P., and Stal, M. (1996). Pattern-oriented Software Architecture: A System of Patterns. John Wiley \& Sons, Inc., New York, NY, USA.

Coad, P. (1992). Object-oriented Patterns. Commun. ACM, 35(9):152-159.

Coplien, J. O. (1992). Advanced C++ Programming Styles and Idioms. Addison-Wesley Longman Publishing Co., Inc., Boston, MA, USA.

Fleiss, J. L. (1981). Statistical methods for rates and proportions. Wiley series in probability and mathematical statistics. Applied probability and statistics. Wiley, cop1981, New York, Chichester, Toronto.

Gamma, E., Helm, R., Johnson, R., and Vlissides, J. (1995). Design Patterns: Elements of Reusable Object-oriented Software. Addison-Wesley Longman Publishing Co., Inc., Boston, MA, USA.

Keegan, D. (2005). The Incorporation of Mobile Learning into Mainstream Education and Training. Proccedings of mLearning20054th World Conference on m-learning, Cape Town.

Kinshuk, T., Suhonen, J., Sutinen, E., and Goh, T. (2003). Mobile technologies in support of distance learning. Asian Journal of Distance Education, v. 1, n. 1, p. $60-68$.

Kitchenham, B. (2004). Procedures for Performing Systematic Reviews. Technical report, Departament of Computer Science, Keele University.

Kitchenham, B. and Charters, S. (2007). Guidelines for performing Systematic Literature Reviews in Software Engineering. Technical Report EBSE 2007-001, Keele University and Durham University Joint Report.

Nah, K. C., White, P., and Sussex, R. (2008). The potential of using a mobile phone to access the internet for learning EFL listening skills within a korean context. ReCALL, Cambridge University Press, New York, NY, USA, v. 20, n. 3, p. 331-347.ISSN 09583440 .

O’Malley, C., Vavoula, G., Glew, J., Taylor, J., and Sharples, M. (2003). Guidelines for learning/teaching/tutoring in a mobile environment.

Quinta, M. R. and de Lucena, F. N. (2010). Problemas e soluções em u-learning e a adaptação de conteúdo de objetos de aprendizagem para diferentes dispositivos. In Anais do 21th Simpósio Brasileiro de Informática na Educação (SBIE 2010).

Svetlana, K. and Yonglk-Yoon (2009). Adaptation e-Learning Contents in Mobile Environment. In Proceedings of the 2Nd International Conference on Interaction Sciences: Information Technology, Culture and Human, ICIS '09, pages 474-479, New York, NY, USA. ACM.

Wexler, S., Brown, J., Metcalf, D., Rogers, D., and Wagner, E. (2008). Mobile learning: What it is, why it matters, and how to incorporate it into your learning strategy. Guild Research. 